\title{
A camera system for IceCube-Gen2
}

\author{
The IceCube-Gen2 Collaboration ${ }^{\dagger}$ \\ ${ }^{\dagger}$ http://icecube.wisc.edu/collaboration/authors/icrc17_gen2 \\ E-mail: mjeong@icecube.wisc.edu, wkang@icecube.wisc.edu
}

\begin{abstract}
The recent discovery of high-energy astrophysical neutrinos and competitive measurements of neutrino oscillation parameters by IceCube motivate a next generation Antarctic neutrino observatory. IceCube-Gen2 is a proposed extension to the current IceCube detector, which uses the extremely clean Antarctic ice as detector medium. While intense calibration efforts with LED flashers have resulted in a good understanding of the ice, ice properties still dominate systematic uncertainties in most analyses. To improve our knowledge of the ice, we propose a novel camera system integrated within the optical sensor modules. The camera system can be installed on all new optical sensor modules to determine their location and orientation and study ice properties. We describe how this system will help us to improve our understanding of the ice. We present a prototype system and simulation that demonstrates its merit.
\end{abstract}

Corresponding authors: M. Jeong ${ }^{* 1}$, W. Kang ${ }^{1}$

${ }^{1}$ Department of Physics, Sungkyunkwan University, Seobu 16419, Suwon, South Korea

35th International Cosmic Ray Conference - ICRC2017

10-20 July, 2017

Bexco, Busan, Korea

${ }^{*}$ Speaker. 


\section{Introduction}

The recent discovery of high-energy astrophysical neutrinos by IceCube [1,2] as well as other results like the competitive measurement of neutrino oscillation parameters [3] have led to studies on possible extensions to the IceCube detector. IceCube-Gen2 [4] is a planned extension to IceCube with an increased detector volume of several cubic-kilometers and a new in-fill array in the current IceCube/DeepCore volume. The upgrade is based on the well proven design concept of IceCube and re-uses its fundamental components.

In IceCube a multitude of calibration devices including two bright calibration light sources called the Standard Candles, retrievable laser systems in the drill holes [5], and LED flashers have resulted in a detailed understanding of the optical properties of the Antarctic ice [6, 7]. The LED flashers, which are twelve radially outward pointing $405 \mathrm{~nm}$ LEDs located on each Digital Optical Module (DOM), are indispensable for the most advanced models of ice properties, as they provide calibration points throughout the detector [6]. Despite successful calibration efforts, the understanding of our detector medium can still be refined. In particular a detailed measurement of the refrozen ice in the drill hole promises improvements [8].

In 2010, two special devices, known as Swedish cameras, were deployed below the deepest DOM of IceCube string 80. They are housed in separate glass spheres $5.8 \mathrm{~m}$ apart at a depth of $\sim 2455 \mathrm{~m}$ [7]. The cameras locally monitored the freeze-in process of the string and observed the formation of the refrozen hole ice. The system found unexpected dust deposits and that the refrozen drill hole contains a central, small region of short scattering length, referred to as bubble column. Motivated by the success of the Swedish camera system, we propose a similar but low-cost system to be directly integrated with future optical sensor modules. The main goal of the new system is to add survey capabilities to our sensor modules and to perform ice property measurements. There is good reason to expect that the environments around each DOM could differ significantly, for example resulting in a non-uniform azimuthal acceptance. With our proposed system the local ice environment can be determined. We introduce the objectives for our camera system in section 2 and then describe simulations to determine camera specifications in section 3. In section 4 we discuss design criteria and prototypes before concluding in section 5 .

\section{Camera System Objectives}

The camera system is expected to conduct quantitative measurements of the ice properties and to deliver qualitative survey information that can be critical to interpret other calibration measurements. It is expected to have a large variety of applications, their relative importance is set by maximizing complementarity to other calibration methods with the overall goal to improve event reconstruction and to reduce ice related systematic uncertainties in analyses. Figure 1 provides an overview of potential measurements and their relative importance. As cameras and LEDs will not be absolutely calibrated the measurements need to rely on relative quantities.

\subsection{Measurement of optical properties of the ice}

The properties of the bulk ice, the undisturbed Antarctic ice, can be determined by observing the shape of the scattering halo of LEDs on adjacent strings. Absorption length and anisotropy 

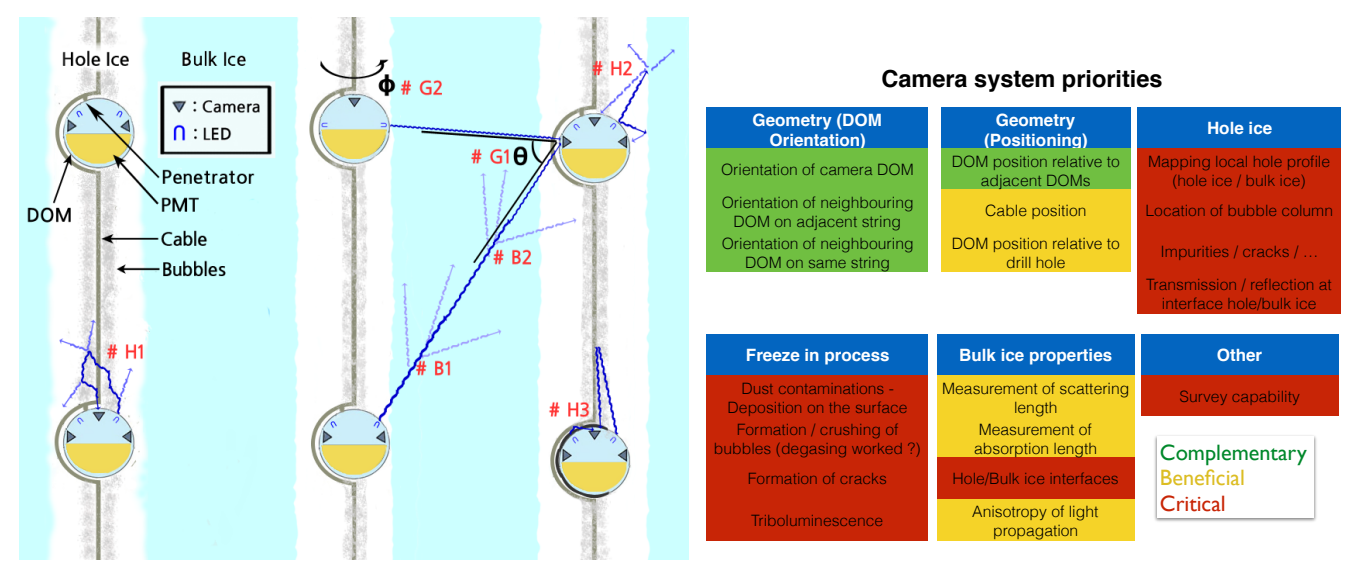

Figure 1: Schematic drawing to illustrate potential camera measurements including geometry $(\# G)$, hole ice $(\# H)$, and bulk ice $(\# B)$ are shown on the left. Relative priorities based on uniqueness of the measurement and complementarity to standard calibration measurements are shown on the right.

might be measurable by comparing the collective image from multiple cameras and LEDs. The hole and bulk ice interface could be observed in reflected light from LEDs and cameras on the same sensor. The measurement might be aided by comparing in-ice with in-water data.

\subsection{Geometry Calibration}

Deployment records are combined with LED flasher measurements in ice [7] to determine the position and orientation of the IceCube DOMs with high precision. Cameras could complement these well established geometry calibration methods and improve them or reduce calibration time. The main benefit of the cameras is expected to come from determining the positioning of the sensors within the drill hole.

\subsection{In-water operation and Freeze-in process}

The cameras can monitor the formation of ice and detect impurities. The system can provide fast feedback if degassing and filtering of drill water, which has been proposed for future drill operations, is effective. In-water data could be critical to understand hole ice formation and provide reference data to in-ice measurements at later times. While $98 \%$ of IceCube DOMs are working well, past failures have almost exclusively occurred during the freeze-in and deployment phase, hence camera operations in water would have to proceed with extreme caution.

\subsection{Hole ice measurements}

Cameras are expected to map the hole ice shape and to determine DOM positions relative to the hole ice walls. The position and extent of the bubble column can be determined and impurities can be located. The hole and bulk ice interface is expected to be visible from reflected light of LEDs within the same hole. However, images from the Swedish Camera suggest that this interface is not very prominent. 


\section{Camera simulations}

To determine the camera hardware requirements and the optimal positioning of the LEDs and cameras, we run simulations and conduct image data analyses. Modeling realistic glacial ice, we determine camera requirements on angular resolution, sensitivity, and LED brightness and wavelength requirements to conduct the proposed measurements. We first introduce the basic simulation and image reconstruction before focusing on one example camera measurement, which was studied in great detail with our simulations. Additional simulations are on-going.

\subsection{Photon propagation simulation and expected camera images}

The photon propagating Monte Carlo simulation is designed to emit photons from LEDs and propagate them in the ice using IceCube's SPICE ice model $[6,10]$, derived from previous calibration studies. Cameras are simulated using the arrival direction of the photons received on a predefined surface area of the sensor modules. Oversizing of the light sensitive area is used to increase statistics. Camera and LED geometries are adjusted for the simulation measurements.

We inject photons following an LED emission profile and record the light received on the surface area of adjacent DOMs. In our simulation DOMs are spaced $17 \mathrm{~m}$ apart on the same string. The distance to adjacent strings is set to $20 \mathrm{~m}$. Figure 2 shows the geometry setup and the received photons on the DOMs surfaces.

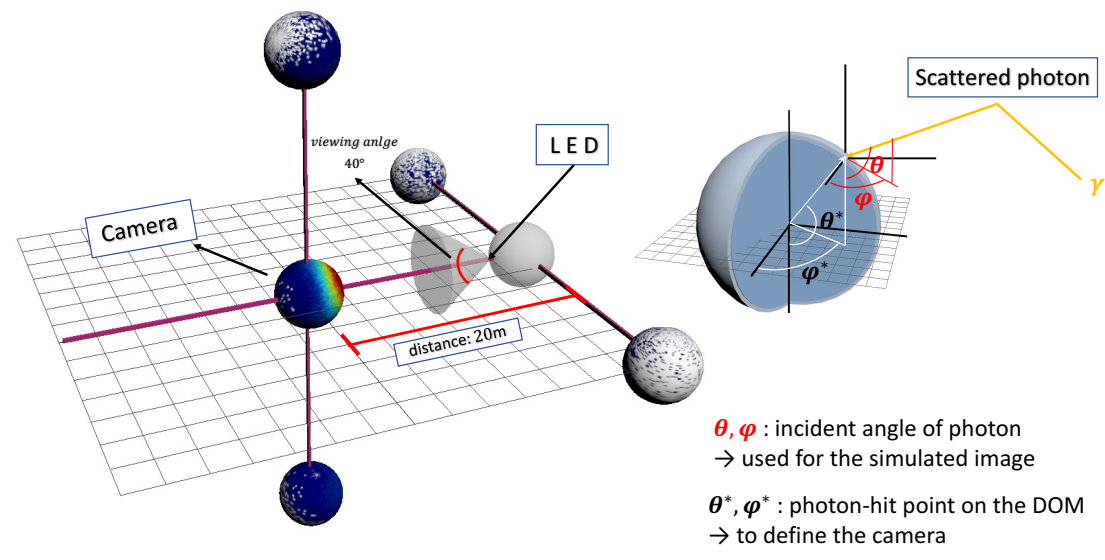

Figure 2: Left: Schematic view of simulation geometry. The DOM on the center right is emitting photons with a specific emission profile, visualized as a white cone. The DOM surfaces display the number of received photons on the point of the surface, varying from blue (small) to red (large). White indicates no counts. Right: $\theta^{\prime}$ and $\phi^{\prime}$ define positions on the DOM surface and are used to specify the camera area; $\theta$ and $\phi$ define the photon arrival direction of a photon $\gamma$. A camera image is given by the recorded $\theta$ and $\phi$ of all photons detected within a small camera area $\theta^{\prime}$ and $\phi^{\prime}$. In yellow a scattered photon $\gamma$ is shown.

In our simulation the entire DOM surface is photo sensitive with $100 \%$ efficiency. Incident angles of arriving photons are recorded and allow us to construct an expected camera image by defining a small surface area on the DOM. Our expected camera image consists just of the arrival azimuth and zenith directions of the photons over a small surface area of the DOM. The surface 
area is kept small to avoid image distortion. We refrain from modeling a camera lens and sensor as it just represents a transformation of the photon arrival directions. A representative image from our simulation is shown in Figure 3. For the simulated camera image we use photon counts, in reality these would correspond to pixel amplitude values, neglecting noise.
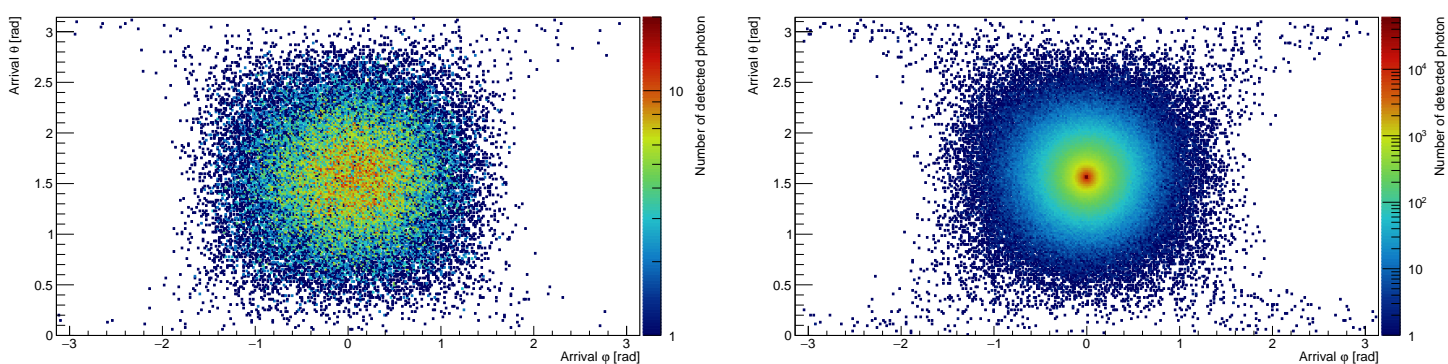

Figure 3: Simulated camera images for a camera observing an LED with $40^{\circ}$ half-opening angle $20 \mathrm{~m}$ away. The left figure is for a short effective scattering length $\left(\lambda_{e}=5.0 \mathrm{~m}\right)$, which shows high-diffusion of photons. In contrast the right figure with a long effective scattering length $\left(\lambda_{e}=77.6 \mathrm{~m}\right)$ shows a more 'point source'-like result.

One relatively straight forward analysis to demonstrate how our simulations inform the design of the camera system is described in the following where idealized conditions to isolate individual ice property effects were used. A real data analysis with multiple ice effects present in an image could prove more complex.

\subsection{Measurement of the scattering length in bulk ice}

We perform an analysis to determine the sensitivity for measuring the scattering length in bulk ice. Two sensor modules are located $20 \mathrm{~m}$ apart at the same depth and a camera is directly looking at an LED that emits light following an emission profile with a $40^{\circ}$ half cone opening angle. For each simulation $2.25 \times 10^{10}$ photons are injected at a monochromatic wavelength of $405 \mathrm{~nm}$. The effective scattering lengths, $\lambda_{e}$, are varied between $5 \mathrm{~m}$ and $77 \mathrm{~m}$. Figure 3 shows the difference between the observed image for a short scattering length (left) and a long one (right). For the short scattering length a well-diffused circular shaped distribution is seen, while the long scattering length results in a 'point source'-like image with a small halo.

While images in Figure 3 qualitatively look very different we develop an image analysis that will be able to quantify the sensitivity for different scattering lengths in the bulk ice. As cameras and LEDs will not be absolutely calibrated we do not use absolute photon counts but only focus on the shape of the scattering halo. The cumulative distribution of the number of photons within a certain radius from the image center (location of the LED) is plotted and normalized (see Figure 4).

Figure 4 further shows that most of the image information is contained within a half-cone opening angle of $40^{\circ}$, which can be interpreted as minimum required FOV. Given that LEDs and cameras will not be aligned in the ice, multiple LEDs and wide field of view cameras are preferred.

\section{System Design and Prototypes}

After summarizing the basic design requirements we introduce our camera prototype system. We have to satisfy engineering requirements, while aiming for an optimal camera design based on 

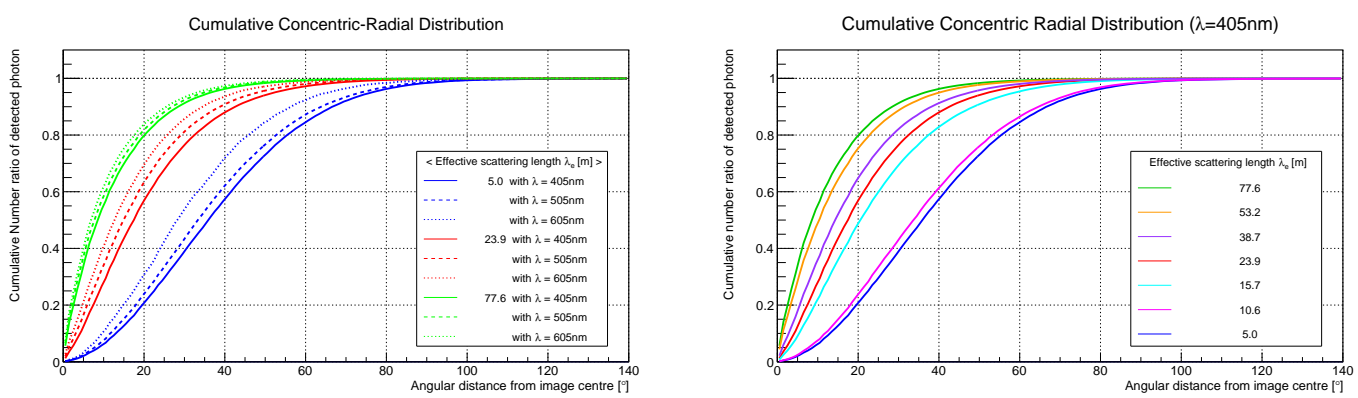

Figure 4: The cumulative photon hits as function of the angular distance from the light source centre for different scattering lengths and wavelengths. The ratio is with respect to all photons detected.

simulations, expected performance, and stability.

For maximal flexibility we propose a system of multiple cameras connected to a controller board $(9.0 \mathrm{~cm} \times 9.0 \mathrm{~cm})$, which then interfaces to the DOM. Camera boards are $3.5 \mathrm{~cm} \times 3.5 \mathrm{~cm}$ in size. In the ice the camera system is expected to operate at temperatures between $-40^{\circ} \mathrm{C}$ and $-20^{\circ} \mathrm{C}$. Cameras will be tested down to $-50^{\circ} \mathrm{C}$. The maximum power consumption of the camera system needs to be kept below $2 \mathrm{~W}$. The system will have no moving parts (fixed focus cameras) for maximal reliability and the number of parts is kept minimal for easy assembly. The system could be complemented with some manual cameras to allow for focus pulling.

To conduct ice property studies, cameras need to be in particular sensitive to observe not only the light coming directly from LEDs, but also scattered light. A large field of view (FOV) is preferred for the cameras to observe multiple neighboring strings. Note that the FOV needs to be adjusted for refraction in the ice. High camera resolution is not required, but could be beneficial for hole ice surveys or the identification of contaminants.

The camera system is designed to be an add-on to the DOM mainboard, as shown in Figure 5. A controller board with processor, RAM and flash memory will control up to three camera modules and communicate with the DOM mainboard. Cameras are connected via flat-flexible cables, which makes their positioning easier.

Prototypes of the camera system have been manufactured using CMOS and CCD

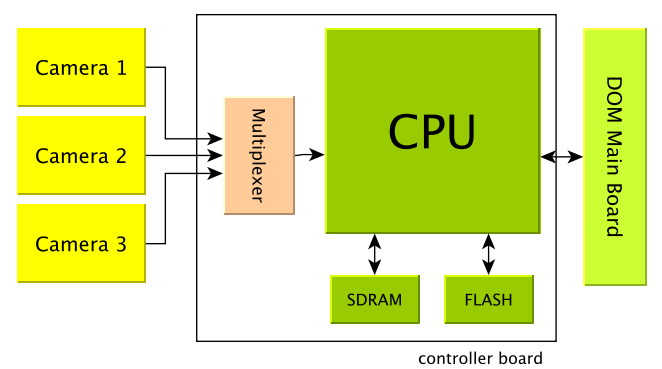

Figure 5: Structure of the camera system. image sensors, respectively. The prototypes are functional and are currently undergoing detailed lab testing.

The prototype of the CMOS camera uses the IMX225LQR-C image sensor from Sony. The sensor has 1.3 million pixels. Its pixels are able to measure the incoming light intensity with a 12 bit dynamic range. The sensor generates output image data through either a CMOS parallel interface, a LVDS or a MIPI interface, which makes it easy for the sensor to be controlled with a wide range of processors. The IMX225LQR-C image sensor is one of the most sensitive image 

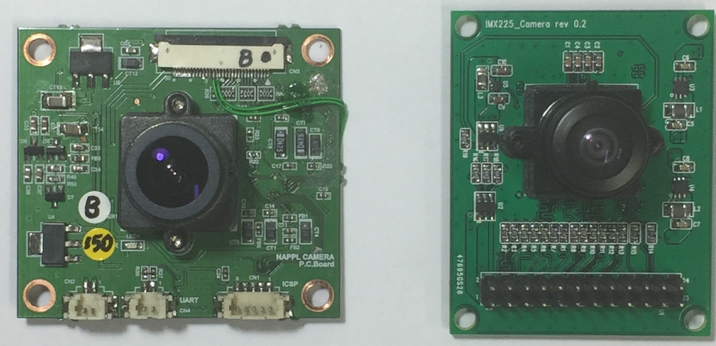

Figure 6: Left: A prototype of the CCD camera module built with the RJ33J3CA0DT image sensor from Sharp. The module is compatible with standard M12-mount lenses and can be connected to a controller board via the CSI connector. Right: A prototype of the CMOS camera module. It contains the IMX225LQR-C image sensor from Sony and is compatible with the M12-mount lenses. In the next version its $2 \times 24$ pin header will be replaced with a CSI connector.

sensors produced by Sony. From laboratory measurements we found that its sensitivity is superior to CMOS image sensors from other manufacturers.

The prototypes of the CCD camera module are built with the RJ33J3CA0DT and RJ33J4CA0DT image sensors from Sharp. They have the same specifications except that the former has color filters but the latter does not. They have the same number of pixels, pixel size, and diagonal length as the IMX225LQR-C sensor. The selection of the image sensors and the design of the camera prototype are made in order to compare the performance of the CMOS image sensor to CCD image sensors with similar specifications. Figure 6 shows the prototypes.

The prototype of the controller is built with a I.MX6Solo Microprocessor Unit (MPU) with an ARM Cortex-A9 CPU. The board contains 512MB of RAM and 8GB of flash memory. This

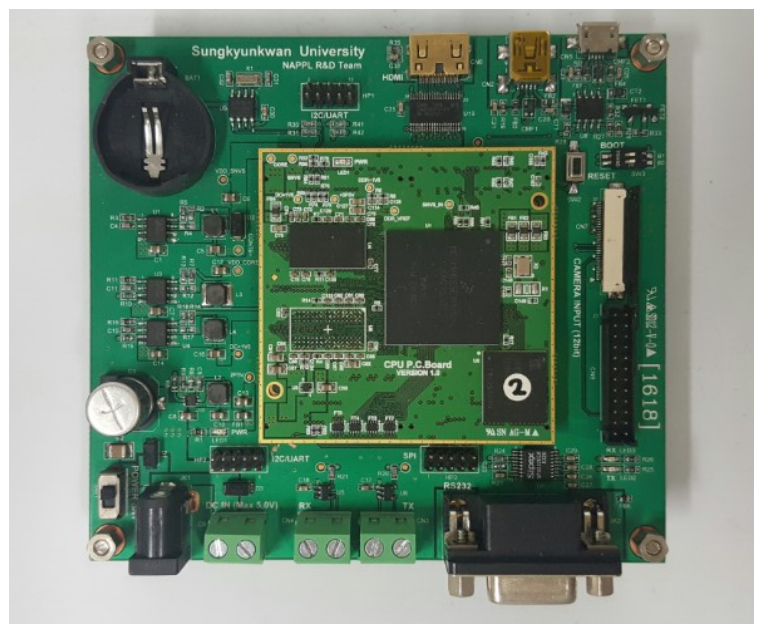

Figure 7: Prototype of camera controller board (rev 1). The rev 1 board is designed to evaluate functionalities of various camera modules. The board contains I.MX6Solo MPU, 512MB DDR3 RAM and 8GB eMMC. For evaluation purpose it includes a HDMI connector, a real time clock and a battery connector as well which will be removed in the next reversion. 
board has enough performance to host both the CMOS camera and the CCD camera prototypes. The prototype of the controller board is shown in Figure 7. It includes additional connections for diagnostics and does not yet have a multiplexer. Therefore, for now only one camera module can be connected. After board evaluation a miniaturized revision will be prepared.

In the ice cameras will only be operated during camera calibration runs. In IceCube, four neighboring DOMs on a string share a common power allocation. To reduce the total power consumption of the camera systems only one camera system on each quad of DOMs would be operated at a time.

\section{Conclusion}

The proposed camera system allows for quantitative measurements of the optical properties of the hole ice, bulk ice and their interface, as well as the detector geometry. The qualitative informations from those measurements can be beneficial to interpret other calibration measurements. Furthermore, the system can be useful to monitor the freeze-in process of the drill holes.

The baseline design of the camera system consists of one controller board and three cameras. In order to achieve optimal performance, both CMOS cameras and CCD cameras are under study. Prototypes of the cameras with the two different image sensor types and the controller board have been produced and are being tested. In the near future their design will be revised to reduce power consumption and enable multiplexing.

In our simulations we have demonstrated that cameras can be used to measure ice properties, such as the effective scattering length, and introduced other potential camera measurements. While only qualitative results are shown in these proceedings, we are also focusing on the quantitative analysis to derive numerical results for the ice properties. Analysis to determine the sensitivity requirements, resolution, and field of view of various ice property measurements are in progress.

\section{References}

[1] IceCube Collaboration, M. G. Aartsen et al., Phys. Rev. Lett. 113 (2014) 101101.

[2] IceCube Collaboration, M. G. Aartsen et al., Science 342 (2013) 1242856.

[3] IceCube Collaboration, M. G. Aartsen et al., Phys. Rev. Lett. 111 (2013) 081801.

[4] IceCube-Gen2 Collaboration, PoS ( ICRC2017) 991 (these proceedings).

[5] N. E. Bramall et al., Geophys. Res. Lett. 32 (2005) L21815.

[6] IceCube Collaboration, M. G. Aartsen et al., Nucl Instrum. \& Meth. A711 (2013) 73.

[7] IceCube Collaboration, M. G. Aartsen et al., JINST 12 (2017) P03012.

[8] M. Rongen, EPJ Web of Conferences 116 (2016) 06011.

[9] IceCube-Gen2 Collaboration, PoS ( ICRC2017) 1047 (these proceedings).

[10] IceCube Collaboration, PoS ( ICRC2013) 508 (2014).

[11] IceCube-Gen2 Collaboration, POS ( ICRC2015) 1145 (2016). 